\title{
So kommt man schnell ans Kinder-Pipi
}

\author{
Aus einer pädiatrischen Ambulanz in Australien kommt ein sehr praktischer Tipp, wie man bei \\ Kleinkindern rasch an Urin kommt. Das Geheimnis ist ein Tupfer mit kalter Kochsalzlösung.
}

_Etwa 5-7\% der fiebernden Kinder unter zwei Jahren haben einen Harnwegsinfekt. Die Diagnose steht und fällt mit dem Urinbefund - doch lassen Kleinkinder einen oft lange auf dieses Material warten. Gerade in der Hektik einer Ambulanz sitzt den Ärzten daher der Katheter oft locker.

Eine Zufallsbeobachtung führte in Australien nun zur systematischen Untersuchung einer neuen Methode: „Quick-Wee“. Dafür randomisierte man 354 Kinder im Alter von 1-12 Monaten in zwei Gruppen. In der einen wurde der Urin normal gewonnen, in der anderen stimulierte man zuvor die suprapubische Region mit einem in kalter Kochsalzlösung getränkten Tupfer.

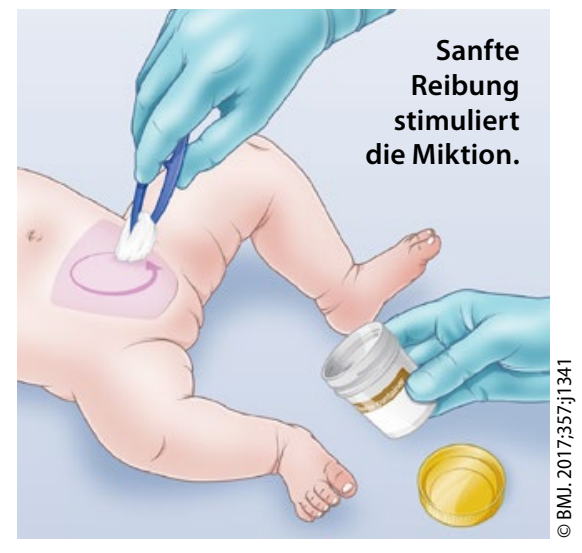

Mit „Quick-Wee“ urinierten 31\% der Kinder innerhalb von fünf Minuten, ohne nur $12 \%$. Noch größer war der Unterschied bei der erfolgreichen Urin- sammlung im sterilen Gefäß. In der Studiengruppe gelang dies in 30\% der Fälle, in der Kontrollgruppe nur in $9 \%$. Oft war man eben mit dem Gefäß nicht schnell genug zur Hand. In der Kontrollgruppe mussten wegen der langen Wartezeit auch die Eltern helfen.

- Kaufman J, Fitzpatrick P, TosifS et al. Faster clean catch urine collection (Quick-Wee method) from infants: randomised controlled trial. BMJ. 2017;357:j1341

\section{KOMMENTAR}

Das lieben wir - wenn am Montag nach dem Kongress etwas in der täglichen Praxisroutine geändert werden muss, weil die Ergebnisse so überzeugend und unmittelbar effektiv sind.

Prof. Dr. med. H. S. FüeßI

\section{Kaposi-Sarkom des harten Gaumens}

Ein 33-jähriger Mann litt seit Monaten unter allgemeiner Müdigkeit und Leistungsschwäche. Außerdem störten ihn ausgedehnte Veränderungen an der Haut und in der Mundhöhle. Bei der Untersuchung erkannte man multiple, derbe, nicht druckdolente, livide Plaques am harten Gaumen (Abb.). Ähnliche Veränderungen fanden sich auch im Gesicht, an der Thoraxwand und an den Beinen. Im Labor fielen eine ausgeprägte Leukopenie mit 2.900 Zellen/ $\mu \mathrm{l}$ und eine CD4-Zellzahl von 76/ $\mu$ l auf. Unter der Diagnose einer HIVInfektion entnahm man Biopsien aus der Haut und den Läsionen in der Mundhöhle, die eine spindelzellartige Neoplasie mit positiver Expression von CD34 und humanem Herpesvirus 8 (HHV-8) bei der immunhistochemischen Testung zeigten. Diese Befunde sprachen für ein Kaposi-Sarkom. Man begann eine antiretrovirale Therapie (ART) und eine Chemotherapie.

Das Kaposi-Sarkom ist eine Neoplasie, die praktisch nur bei Patienten mit ausgeprägtem zellulärem Immundefekt vorkommt. Ausgelöst wird es durch HHV-8. Es kann isoliert auf der Haut auftreten, aber auch innere Organe befallen, vor allem die Mundhöhle, den Gastrointestinal- und den Respirationstrakt.

Ein derart ausgedehnter Befund wie im vorliegenden Fall ist mit einer schlechten Prognose assoziiert. Dennoch ging die Tumorgröße bei dem Patienten nach mehreren Monaten Therapie zurück.

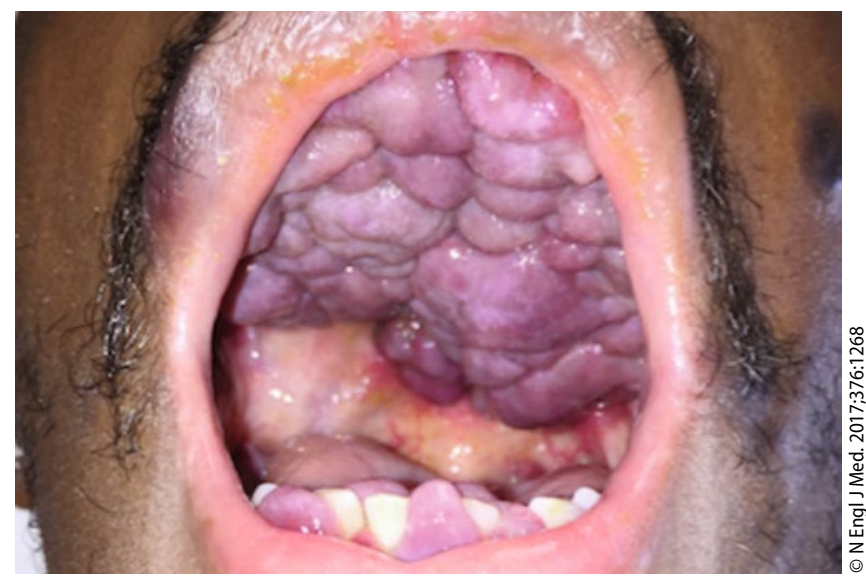

Derbe, livide Plaques am harten Gaumen.

Unter fortlaufender ART stieg die CD4-Zellzahl an, und der Patient fühlte sich besser.

Prof. Dr. med. H. S. FüeßI

- Kennedy-LeJeune E, Cataldo VD. Kaposi's sarcoma of the oral cavity. N Engl J Med. 2017;376:1268 